\title{
Impactos da pandemia da Covid-19 no autocuidado de idosos: Uma revisão
}

\section{integrativa}

\section{Impacts of the Covid-19 pandemic on elderly self-care: An integrative review}

Impactos de la pandemia del Covid-19 en el cuidado personal de Los ancianos: Una revisión

\author{
integrativa
}

Eduardo Odonete Marques ORCID: https://orcid.org/0000-0002-4739-6474

Universidade Federal do Piauí, Brasil

E-mail: eduardomarques@ufpi.edu.br

Sandryelle de Andrade Rodrigues ORCID: https://orcid.org/0000-0002-0878-3885

Centro Universitário Doutor Leão Sampaio, Brasil

E-mail: sandryellerodrigues24@gmail.com

Milena Cordeiro de Freitas

ORCID: https://orcid.org/0000-0003-0208-9400

Faculdade Cearense, Brasil

E-mail: barra.milenafreitas@hotmail.com

Thayane Cintra Lemos

ORCID: https://orcid.org/0000-0002-8918-7531

Universidade Federal do Espirito Santo, Brasil

E-mail: thayane309@gmail.com

Vitória Luiza Cavalcanti de Lima

ORCID: https://orcid.org/0000-0002-1311-2832

Faculdade Cearense, Brasil

E-mail: vit_oria_cavalcanti@hotmail.com

Paula Ermans de Oliveira

ORCID: https://orcid.org/0000-0002-2735-5202

Universidade Portiguar, Brasil

E-mail: paulaaermans@gmail.com

Kamila de Castro Morais

ORCID: https://orcid.org/0000-0002-3564-7993

Universidade Regional do Cariri, Brasil

E-mail: kamilacastromorais@gmail.com

Daniel Coutinho dos Santos

ORCID: https://orcid.org/0000-0002-6230-9842

Universidade Federal do Maranhão, Brasil

E-mail: daniel.coutinho@discente.ufma.br

André Sousa Rocha

ORCID: https://orcid.org/0000-0002-0185-9699 Universidade São Francisco, Brasil

E-mail: andresousarocha9@gmail.com

Antônio Diego Costa Bezerra

ORCID: https://orcid.org/0000-0002-2441-2961

Centro Universitário Unifanor, Brasil Fundação Oswaldo Cruz, Brasil

E-mail: diegocostamjc@gmail.com

Cidianna Emanuelly Melo do Nascimento

ORCID: https://orcid.org/0000-0001-5477-4413

Universidade Estadual do Ceará, Brasil

E-mail: profa.cidianna.melo@gmail.com

\section{Resumo}

Introdução: A população idosa possui maior susceptibilidade à pandemia da COVID-19 (SARS-CoV-2). Torna-se de suma importância, a prática do autocuidado, em prol da preservação da vida e da saúde. Objetivo: Analisar na literatura os impactos da pandemia da COVID-19 no autocuidado de idosos. Método: Para esta revisão integrativa da literatura, utilizou-se a associação de dois descritores controlados: Autocuidado (Self Care), Idoso (Aged) e um não controlado: COVID-19 (COVID-19). A pesquisa ocorreu nas bases eletrônicas: Medline, Web of Science e Scopus. Resultou-se em 18 artigos, publicados entre 2020 e 2021, selecionados de acordo com os critérios de inclusão e exclusão previamente estabelecidos. Resultados: O isolamento social possibilita fatores que interferem no autocuidado de idosos, como 
depressão, surgimento ou agravamento de doenças crônicas, como diabetes e doenças cardiovasculares, tornando-os mais susceptíveis ao contágio da doença. Pesquisas relatam que idosos mais jovens, possuem melhor compreensão dos riscos da COVID-19 e praticam o autocuidado com maior frequência. Todavia, o autocuidado juntamente com o apoio social, o uso da tecnologia e a prática de atividades físicas são importantes ferramentas de intervenção. Considerações finais: Portanto, a pandemia resultou em impactos no autocuidado de idosos gerando efeitos maléficos a saúde física e mental, devido ao isolamento social.

Palavras-chave: Autocuidado; COVID-19; Idoso.

\begin{abstract}
Introduction: The elderly population is more susceptible to the COVID-19 (SARS-CoV-2) pandemic. It becomes of paramount importance, the practice of self-care, in favor of the preservation of life and health. Objective: To analyze in the literature the impacts of the COVID-19 pandemic on the self-care of the elderly. Method: For this integrative review of the literature, the association of two controlled descriptors was used: Self-Care (Self Care), Elderly (Aged) and an uncontrolled one: COVID-19 (COVID-19). The research took place in the electronic databases: Medline, Web of Science and Scopus. It resulted in 18 articles, published between 2020 and 2021, selected according to the inclusion and exclusion criteria previously established. Results: Social isolation allows factors that interfere with the self-care of the elderly, such as depression, the onset or worsening of chronic diseases, such as diabetes and cardiovascular diseases, making them more susceptible to the contagion of the disease. Research reports that younger elderly people have a better understanding of the risks of COVID-19 and practice self-care more frequently. However, self-care together with social support, the use of technology and the practice of physical activities are important intervention tools. Final considerations: Therefore, the pandemic resulted in impacts on the self-care of the elderly, generating harmful effects on physical and mental health, due to social isolation.
\end{abstract}

Keywords: Self-care; COVID-19; Elderly.

\title{
Resumen
}

Introducción: la población anciana es más susceptible a la pandemia COVID-19 (SARS-CoV-2). Se vuelve de suma importancia, la práctica del autocuidado, a favor de la preservación de la vida y la salud. Objetivo: Analizar en la literatura los impactos de la pandemia COVID-19 en el autocuidado del adulto mayor. Método: Para esta revisión integradora de la literatura se utilizó la asociación de dos descriptores controlados: Autocuidado (Autocuidado), Anciano (Anciano) y uno no controlado: COVID-19 (COVID-19). La investigación se realizó en las bases de datos electrónicas: Medline, Web of Science y Scopus. Resultó en 18 artículos, publicados entre 2020 y 2021, seleccionados según los criterios de inclusión y exclusión previamente establecidos. Resultados: El aislamiento social permite factores que interfieren en el autocuidado de los ancianos, como la depresión, la aparición o agravamiento de enfermedades crónicas, como la diabetes y las enfermedades cardiovasculares, haciéndolos más susceptibles al contagio de la enfermedad. Las investigaciones informan que las personas mayores más jóvenes comprenden mejor los riesgos del COVID-19 y practican el autocuidado con más frecuencia. Sin embargo, el autocuidado junto con el apoyo social, el uso de la tecnología y la práctica de actividades físicas son importantes herramientas de intervención. Consideraciones finales: Por tanto, la pandemia generó impactos en el autocuidado de las personas mayores, generando efectos nocivos en la salud física y mental, debido al aislamiento social.

Palabras clave: Autocuidado; COVID-19; Anciano.

\section{Introdução}

A pandemia da COVID-19, doença causada pelo novo coronavírus (SARS-CoV-2), levou os indivíduos ao isolamento social, limitando e afetando de forma negativa a qualidade de vida de idosos, ocasionando problemas respiratórios agudos graves além de manifestações digestivas e sistêmicas, dessa forma, prejudicando a saúde mental e física, gerando o aumento de casos de depressão, ansiedade e doenças crônicas (Nancy et al., 2020).

Diante da rápida disseminação mundial da COVID-19 e visando diminuir seu contágio, a Organização Mundial da Saúde (OMS) recomenda medidas preventivas, como distanciamento social, para minimizar o risco do contágio. Todavia, a manutenção de bem-estar físico e psicológico, além de estilos de vida saudáveis são importantes para manutenção e qualidade de vida (Maria et al., 2020).

De acordo com Sun et al. (2020), idosos que realizam o autocuidado conseguem reconhecer sintomas e elaborar estratégias para desempenhar atividades que promovem a melhoria da saúde e condições de vida. Ademais, a população idosa possui maior susceptibilidade à infecção por Sars-COV-2, em decorrência da carência imunológica, tornando ainda mais importante a manutenção do autocuidado por esse grupo. 
Deste modo, para o desempenho do autocuidado, o idoso necessita de condições favoráveis para realização de atividades do cotidiano. Para isso, é relevante fortalecer a importância de um envelhecer saudável diante contexto pandêmico do novo coronavírus (Coutinho \& Tomasi, 2020). Diante disso, o estudo foi norteado pela seguinte questão norteadora: Quais os impactos da pandemia da COVID-19 no autocuidado de idosos?

Considerando que a população idosa vem crescendo em ritmo acelerado em todo o mundo, percebendo que o cuidado a esse grupo etário deve ser direcionado a profissionais capacitados para ofertar uma assistência eficaz e segura, o estudo tem como objetivo: analisar, segundo a literatura, os impactos da pandemia da COVID-19 no autocuidado de idosos.

\section{Metodologia}

Trata-se de uma revisão bibliográfica integrativa, que se configura em uma metodologia que proporciona a síntese do conhecimento e a incorporação da aplicabilidade de resultados de estudos significativos na prática (Souza, Silva \& Carvalho, 2010). Por meio da pergunta norteadora, realizou-se análises de pesquisas existentes, para formação de um arcabouço e dessa forma a compilação de resultados, que garantam um estudo aprofundado do tema de maneira sistemática e organizada. Por se tratar de uma revisão integrativa, esse artigo foi construído respeitando as seguintes etapas: Formulação da pergunta norteadora; localização dos estudos; avaliação crítica dos estudos; coleta de dados; análise e apresentação dos dados; interpretação dos dados, aprimoramento e atualização da revisão (Botelho, Cunha \& Macedo, 2011).

A busca e a seleção foram realizadas por meio da utilização dos descritores oficiais, na língua portuguesa e inglesa, sendo dois deles controlados: Autocuidado (Self Care), Idoso (Aged) e um não controlado: COVID-19 (COVID-19), associandoos mediante o operador booleano "and”. A pesquisa ocorreu no mês de fevereiro de 2021, sendo efetuada a partir de um levantamento de dados bibliográficos disponíveis nas bases de dados: Medline, Web of Science e Scopus. Para a seleção das produções científicas, o pesquisador obedeceu aos seguintes critérios de inclusão: o emprego de artigos originais disponíveis na íntegra, on-line, de forma gratuita, com assuntos relevantes à temática em questão, em idiomas português, inglês ou espanhol, publicados entre 2020 e 2021. Os critérios de exclusão foram: publicações duplicadas, dissertações, teses, editoriais e fatores que não satisfazem na estratégia PICO (Tabela 1).

Tabela 1 - Descrição da estratégia PICO.

\begin{tabular}{ccc}
\hline Iniciais & Descrição & Análise \\
\hline $\mathrm{P}$ & Paciente / problema & Idosos diante o impacto da pandemia do novo coronavírus \\
$\mathrm{I}$ & Intervenção & Autocuidado em meio quarentena \\
$\mathrm{C}$ & $\begin{array}{c}\text { Comparação / } \\
\text { controle } \\
\end{array}$ & Resultado / desfecho jovens \\
O & Melhora ou influência por meio do autocuidado na qualidade de vida em meio contexto \\
\end{tabular}

Fonte: Autores (2021).

Como fundamento de pesquisa a estratégia PICO representa um acrônimo: P - paciente/problema; I - intervenção; C comparação/controle; O - resultado/desfecho. Sendo esta, uma importante ferramenta utilizada pela prática baseada em evidências científicas, para auxílio do levantamento bibliográfico buscando solucionar problemas da prática assistencial, de ensino e pesquisa (Santos, Pimenta \& Nobre, 2007). 
Identificou-se no total 366 estudos usando os descritores (Self Care and Covid-19 and Aged) nas respectivas bases de dados, apresentados na Tabela 2, conforme os critérios e as estratégias de busca utilizadas.

Tabela 2 - Número de estudos identificados nas bases de dados.

\begin{tabular}{lccc}
\hline & Total de estudos encontrados & Estudos selecionados & Estudos excluídos após leitura \\
\hline Medline & 57 & 6 & 51 \\
$\begin{array}{l}\text { Web of } \\
\text { Science }\end{array}$ & 204 & 8 & 196 \\
Scopus & 105 & 4 & 101 \\
Total & 366 & 18 & 348 \\
\hline
\end{tabular}

Fonte: Autores (2021).

Por meio da pesquisa, qualitativa, com o auxílio do processo de interpretação e compreensão do assunto estudado onde são extremamente úteis para "descobrir" aspectos novos de um problema, o seu significado e a relevância diante a realidade (Ludke \& Andre, 2013). Após a avaliação crítica de 366 estudos pré-selecionados, 348 foram excluídos e a presente revisão foi constituída por 18 estudos que se enquadram nas normas de elegibilidade conforme demonstrado no acrônimo PICO (Tabela1).

\section{Resultados e Discussão}

Nesta revisão foram selecionados 18 artigos, indexados entre os anos de 2020 e 2021, sendo dezesseis (88,88\%) de 2020 e dois $(11,1 \%)$ de 2021. Foi elaborada uma tabela, descrevendo a quantidade e porcentagem de artigos selecionados em cada base de dados, bem como o ano de publicação (Tabela 3). Dos estudos analisados, três (16,66\%) foram desenvolvidos nos Estados Unidos, dois $(11,11 \%)$ na China, dois (11,11\%) na Espanha, um (5,55\%) no México, um $(5,55 \%)$ na Itália, um (5,55\%) no Japão, um $(5,55 \%)$ na África, um $(5,55 \%)$ na Noruega, um $(5,55 \%)$ na Dinamarca, um $(5,55 \%)$ no Irã, um $(5,55 \%)$ na Austrália, um (5,55\%) em Gana, um em (5,55\%) Taiwan e um (5,55\%) no Canadá.

Tabela 3 - Descrição das bases, número de artigos selecionados e ano de publicação.

\begin{tabular}{ccc}
\hline Base de dados & Evidências científicas & Ano de publicação \\
\hline Medline & Seis $(33,33 \%)$ & $2020(83,33 \%)$ e $2021(16,66 \%)$ \\
Web of Science & Oito $(44,44 \%)$ & $2020(100 \%)$ \\
Scopus & Quatro $(22,22 \%)$ & $2020(75 \%)$ e $2021(25 \%)$ \\
\hline
\end{tabular}

Fonte: Autores (2021).

A análise dos estudos com o objetivo de identificar os impactos da pandemia da COVID-19 no autocuidado de idosos por meio de uma revisão da literatura, possibilitou a construção de duas categorias: I - Impactos da pandemia da COVID-19 na saúde e autocuidado de idosos em isolamento social e II - Estratégias para melhoria do bem-estar psicológico e qualidade de vida dos idosos. 


\section{Categoria I - Impactos da pandemia da COVID-19 na saúde e autocuidado de idosos em isolamento social}

Durante o período de pandemia, os idosos apresentaram grande probabilidade de desenvolverem ansiedade e depressão, em decorrência do estresse psicológico vivenciado (Chen, 2020). O isolamento social pode aumentar a prevalência de solidão, demência, delírio e suicídio (Mobasseri et al., 2020).

Outrossim, um estudo observou que a preocupação com o futuro e o estresse emocional foram sentimentos relatados com grande frequência pelos idosos no decorrer da pandemia da COVID-19 (Whitehead \& Torossian, 2020). As iniquidades sociais, o desamparo na saúde pública e as condições econômicas impactaram negativamente na saúde da população idosa, tornando-os mais vulneráveis e inseguros (Chen, 2020; Ntsama Essomba et al., 2020).

O confinamento distanciou muitas famílias em prol da minimização dos riscos de contaminação do Sars-COV-2. Com isso, o sentimento de solidão provocou reações negativas em grande parte dos idosos (Monahan et al. 2020). Com o aumento dos sintomas psicológicos, as atividades de autocuidado mostraram-se reduzidas entre muitos idosos, diminuindo o nível de bem-estar e aumentando o nível de estresse percebido (Martínez et al. 2021). Idosos que mantêm escores maiores de autocuidado e apoio social, possuem melhores resultados em termos de qualidade de vida e bem-estar psicológico (Maria et al. 2020; Martinez et al. 2020; Whitehead \& Torossian, 2020).

\section{Categoria II - Estratégias para melhoria do bem-estar psicológico e qualidade de vida dos idosos}

O conceito de resiliência aborda a capacidade do indivíduo de superar crises e obstáculos, adaptar-se a mudanças e resistir às situações estressantes por meio de soluções estratégicas para tal (Yunes, 2003). Com a resiliência e a manutenção do suporte social, os idosos conseguem lidar melhor com dificuldades, retornando ao equilíbrio com mais facilidade. Assim, a capacidade de resiliência durante a pandemia da COVID-19, pode auxiliar na recuperação da saúde física, cognitiva e mental (Lee, 2020; Chen, 2020).

A utilização de tecnologias móveis pode ser uma potente aliada ao envelhecimento saudável, uma vez que esta abre portas para serviços como telemedicina e telecomunicações. Ademais, a interação social por meio da tecnologia pode possibilitar redução da exclusão digital e aproximação do meio familiar de forma intergeracional (Chen, 2020; Morrow- Howell, 2020). Todavia, sabe-se que o uso da tecnologia por idosos ainda possui baixo índice de adesão, seja por condições socioeconômicas e/ou cognitivas limitantes, localização geográfica, sentimento de incapacidade ou até mesmo pouco interesse em aprender (Gorenko et al. 2021).

Ademais, a prática de atividades físicas mostrou contribuir de forma positiva para o envelhecimento ativo, mobilidade, qualidade de vida e autocuidado, além de reduzir o medo e risco de quedas em idosos (Levinger et al., 2020; Rodríguez-González et al., 2020). Contudo, com as atividades ao ar livre limitadas, é preciso buscar alternativas para manter-se em movimento, como por exemplo acompanhamento profissional de forma online por meio de vídeo chamadas para a realização de atividades mais simples em casa. O incentivo familiar auxilia o desempenho ao contribuir para realização do processo, motivando os idosos a realizarem o autocuidado (Chen, 2020; Morrow-Howell, 2020).

\section{Considerações Finais}

As mudanças comportamentais do isolamento social decorrente da pandemia do novo coronavírus gerou impactos negativos no autocuidado de idosos ocasionando efeitos maléficos ao bem-estar físico e mental, como ansiedade e estresse, dessa forma causando o surgimento ou agravamento das doenças crônicas nessa população, como diabetes e doenças cardiovasculares, tornando-os mais susceptíveis ao contágio da COVID-19. Todavia, o apoio social, fé, hobbies, o uso da tecnologia e a prática de atividades físicas juntamente com o autocuidado são importantes ferramentas de intervenção para melhorar a qualidade de vida e bem-estar em meio contexto pandêmico dos idosos. 


\section{Financiamento}

Para a condução desse estudo não houve financiamento por agências de fomento, sendo de inteira responsabilidade dos autores todos os custos oriundos para a construção deste manuscrito.

\section{Conflitos de Interesse}

Os autores declaram que não existem interesses concorrentes.

\section{Referências}

Botelho, L. L. R., Cunha, C. C. de A., \& Macedo, M. (2011). O método da revisão integrativa nos estudos organizacionais. Gestão e Sociedade. 5(11), 121-136.

Coutinho, L. S. B. \& Tomasi, E. (2020). Déficit de autocuidado em idosos: características, fatores associados e recomendações às equipes de estratégia saúde da família. Interface - Comunicação, saúde, educação, 24(1), e190578. https://doi.org/10.1590/interface.190578

Chen, K. (2020). Use of Gerontechnology to Assist Older Adults to Cope with the COVID-19 Pandemic. Journal of the American Medical Directors Association, 21(7), 983-984. https://doi.org/10.1016/j.jamda.2020.05.021

Gorenko, J. A., Moran, C., Flynn, M., Dobson, K., \& Konnert, C. (2021). Social Isolation and Psychological Distress Among Older Adults Related to COVID19: A Narrative Review of Remotely Delivered Interventions and Recommendations. Journal of applied gerontology: the official journal of the Southern Gerontological Society, 40(1), 3-13. https://doi.org/10.1177/0733464820958550

Lee, K., Jeong, G. C., \& Yim, J. (2020). Consideration of the Psychological and Mental Health of the Elderly during COVID-19: A Theoretical Review. International journal of environmental research and public health, 17(21), 8098. https://doi.org/10.3390/ijerph17218098

Levinger, P., Panisset, M., Dunn, J., Haines, T., Dow, B., Batchelor, F., Biddle, S., Duque, G., \& Hill, K. D. (2020). Exercise intervention outdoor project in the community for older people - results from the ENJOY Seniors Exercise Park project translation research in the community. BMC geriatrics, $20(1)$, 446. https://doi.org/10.1186/s12877-020-01824-0

Ludke, M. \& Andre, M. E. D. A. (2013). Pesquisas em educação: uma abordagem qualitativa. E.P.U. 6.

Maria, M., Ferro, F., Ausili, D., Alvaro, R., De Marinis, M. G., Di Mauro, S., Matarese, M., \& Vellone, E. (2020). Development and Psychometric Testing of the Self-Care in COVID-19 (SCOVID) Scale, an Instrument for Measuring Self-Care in the COVID-19 Pandemic. International journal of environmental research and public health, 17(21), 7834. https://doi.org/10.3390/ijerph17217834

Martínez, V., et al (2020). Validity and reliability of the Self-Care Activities Screening Scale (SASS-14) during COVID-19 lockdown. 10.21203/rs.3.rs42014/v1.

Mobasseri, K., Azami-aghdash, S., Khanijahani, A., \& Khodayari-Zarnaq, R. (2020). The Main Issues and Challenges Older Adults Face in the SARS-CoV-2 Pandemic: A Scoping Review of Literature. Iranian Journal of Public Health, 49(12), 2295-2307. https://doi.org/10.18502/ijph.v49i12.4810

Monahan, C., Macdonald, J., Lytle, A., Apriceno, M., \& Levy, S. R. (2020). COVID-19 and ageism: How positive and negative responses impact older adults and society. The American psychologist, 75(7), 887-896. https://doi.org/10.1037/amp0000699

Morrow-Howell, N., Galucia, N., \& Swinford, E. (2020). Recovering from the COVID-19 Pandemic: A Focus on Older Adults. Journal of aging \& social policy, 32(4-5), 526-535. https://doi.org/10.1080/08959420.2020.1759758

Nancy Morrow-Howell, Natalie Galucia \& Emma Swinford. (2020). Recovering from the COVID-19 Pandemic: A Focus on Older Adults. Journal of Aging \& Social Policy, 32: 4-5, 526-535, DOI: 10.1080 / 08959420.2020. 1759758

Ntsama Essomba, M. J., Nzana, V., Noubiap, J. J., Zingui-Ottou, M., Ciaffi, L., Sobngwi, E., \& Ashuntantang, G. (2020). The Follow-Up and Well-Being of Geriatric Outpatients During COVID-19 Pandemic in Cameroon: Insights From the Yaounde Central Hospital. Gerontology \& geriatric medicine, 6, 2333721420959242. https://doi.org/10.1177/2333721420959242

Rodríguez-González, R., Facal, D., Martínez-Santos, A. E., \& Gandoy-Crego, M. (2020). Psychological, Social and Health-Related Challenges in Spanish Older Adults During the Lockdown of the COVID-19 First Wave. Frontiers in psychiatry, 11, 588949. https://doi.org/10.3389/fpsyt.2020.588949

Santos, C. M.; Costa, Pimenta, Cibele Andrucioli de Mattos, \& Nobre, Moacyr Roberto Cuce. (2007). A estratégia PICO para a construção da pergunta de pesquisa e busca de evidências. Revista Latino-Americana de Enfermagem, 15(3), 508-511. https://doi.org/10.1590/S0104-11692007000300023

Sun, Z., Yang, B., Zhang, R., \& Cheng, X. (2020). Influencing Factors of Understanding COVID-19 Risks and Coping Behaviors among the Elderly Population. International journal of environmental research and public health, 17(16), 5889. https://doi.org/10.3390/ijerph17165889

Souza, M. T.; Silva, M. D. \& Carvalho, R. (2010). Revisão integrativa: o que é e como fazer. Einstein, 8(1), 102-106. https://dx.doi.org/10.1590/s167945082010 rw1134

Whitehead, B. R., \& Torossian, E. (2021). Older Adults' Experience of the COVID-19 Pandemic: A Mixed-Methods Analysis of Stresses and Joys. The Gerontologist, 61(1), 36-47. https://doi.org/10.1093/geront/gnaa126 
Research, Society and Development, v. 10, n. 3, e56910313672, 2021 (CC BY 4.0) | ISSN 2525-3409 | DOI: http://dx.doi.org/10.33448/rsd-v10i3.13672

Yunes, M. A. M. (2003). Psicologia positiva e resiliência: o foco no indivíduo e na família. Psicologia em Estudo, 8, 75-84. https://doi.org/10.1590/S141373722003000300010 